$\xi$

\title{
Bipolar Fuzzy Soft Hyperideals and Homomorphism of Gamma-Hypersemigroups
}

\author{
K.Arulmozhi ${ }^{1}$, V.Chinnadurai ${ }^{2}$, M.Seenivasan ${ }^{3}$ \\ ${ }^{1}$ Department of Mathematics, Vels Institute of Science,Technology and Advanced Studies,(VISTAS) Chennai-600117 \\ Department of Mathematics, Annamalai University,Annamalainagar-608002 \\ *Corresponding author E-mail:emseeni@rediffmail.com
}

\begin{abstract}
In this paper, we introduce the concept of bipolar fuzzy soft gamma hyperideals in gamma hyper semigroups. We define bipolar fuzzy soft hyper ideals, bi-ideals and interior ideals of gamma hyper semigroups and discuss some properties.
\end{abstract}

Keywords: Soft set, $\Gamma$ - hyper semigroups, bipolar valued fuzzy set, hyper ideal, homomorphism.

\section{Introduction}

Zadeh [18] introduced the concept of fuzzy sets in 1965. Algebraic hyper structures represent a natural extension of classical algebraic structures, and they were originally proposed in 1934 by Marty [7]. One of the main reasons which attract researchers towards hyperstructures is its unique property that in hyperstructures composition of two elements is a set, while in classical algebraic structures the composition of two elements is an element. Zhang [19] introduced the notion of bipolar fuzzy sets. Lee [4] used the term bipolar fuzzy sets as applied to algebraic structures. Bipolar fuzzy $\Gamma$-hyperideals in $\Gamma$-hyper semigroups was studied by Naveed Yaqoob et al [14]. Soft set theory was introduced by Molodtsov [8] in 1999, and its a new mathematical model for dealing with uncertainty from a parameterization point of view. Maji et al [6] studied the some new operations on fuzzy soft sets. Aygunoglu and Aygun [3] introduced the notion of a fuzzy soft group. The concept of bipolar fuzzy soft sets has been introduced by Naz et al [12] Aslam et al [2] worked on bipolar fuzzy soft sets and their special union and intersection. Bipolar fuzzy soft $\Gamma$-semigroups was introduced by Muhammad Akram et al [10]. $\Gamma$-semigroups was introduced by Sen and Saha [16]. In this paper, we define a new notion of bipolar fuzzy soft $\Gamma$ - hyper semigroups and investigate some of its properties with examples.

\section{Preliminaries}

In this section, we list some basic definitions.

Definition 2.1[16]

Let $S=\{a, b, c, \ldots\}$ and $\{\alpha, \beta, \gamma, \ldots\}$ be two non-empty sets. Then $\mathrm{S}$ is called a $\Gamma$-semigroup if it satisfies the conditions

(i) $a \alpha b \in S$,

(ii) $(\mathrm{a} \beta \mathrm{b}) \gamma \mathrm{c}=\mathrm{a} \beta(\mathrm{b} \gamma \mathrm{c}) \forall \mathrm{a}, \mathrm{b}, \mathrm{c} \in \mathrm{S}$ and $\alpha, \beta, \gamma \in \Gamma$.

Definition 2.2

A map $\circ: \mathrm{H} \times \mathrm{H} \rightarrow \mathrm{P}^{*}(\mathrm{H})$ is called a hyper operation or join operation on the set $\mathrm{H}$, where $\mathrm{H}$ is a non-empty set and $\mathrm{P}^{*}(\mathrm{H})=$ $\mathrm{P}(\mathrm{H}) \backslash\{\phi\}$ denotes the set of all non-empty subset of $\mathrm{H}$. A

hypergroupoid is a set $\mathrm{H}$ together with a (binary) hyperoperation.

\section{Definition 2.3}

A hypergroupoid $(\mathrm{H}, \circ)$, which is associative, that is $\mathrm{x} \circ(\mathrm{y} \circ \mathrm{z})=$ $(\mathrm{x} \circ \mathrm{y}) \circ \mathrm{z}$ for all $\mathrm{x}, \mathrm{y}, \mathrm{z} \in \mathrm{H}$, is called a hyper semigroup. Let $\mathrm{A}$ and $\mathrm{B}$ be two non-empty subsets of $\mathrm{H}$. Then we define

$$
A \circ B= \begin{cases}\bigcup_{\mathrm{a} \in \mathrm{A}, \mathrm{b} \in \mathrm{B}} \mathrm{a} \circ \mathrm{b}, & \mathrm{a} \circ \mathrm{B}=\{\mathrm{a}\} \circ \mathrm{B} \\ \mathrm{A} \circ \mathrm{b}=\mathrm{A} \circ\{\mathrm{b}\}\end{cases}
$$

Definition 2.4[1]

Let $S$ and $\Gamma$ be two non-empty sets. $S$ is called a $\Gamma$-hypersemigroup if every $\gamma \in \Gamma$ is a hyperoperation on $S$ that is $\mathrm{x} \gamma \mathrm{y} \subseteq \mathrm{S}$ for every $\mathrm{x}, \mathrm{y} \in \mathrm{S}$, and for every $\alpha, \beta \in \Gamma$ and $\mathrm{x}, \mathrm{y}, \mathrm{z} \in \mathrm{H}$ we have $x \alpha(y \beta z)=(x \alpha y) \beta z$. If every $\gamma \in \Gamma$ is a hyper operation, then $S$ is a $\Gamma$-semigroup. If $(S, \gamma)$ is a hypergroup for every $\gamma \in \Gamma$, then $S$ is called a $\Gamma$-hypergroup. Let $A$ and $B$ be two non-empty subsets of $S$ and $\gamma \in \Gamma$. We define $A \gamma B=U\{a \gamma b \mid a \in A, b \in B\}$. Also $\mathrm{A} \Gamma \mathrm{B}=U\{\mathrm{a} \gamma \mathrm{b} \mid \mathrm{a} \in \mathrm{A}, \mathrm{b} \in \mathrm{B}$ and $\gamma \Gamma\}=\bigcup_{\mathrm{A} \gamma \mathrm{B}}$. Let $\mathrm{S}$ be $\mathrm{a}$ $\Gamma$-hypersemigroup and let $\gamma \in \Gamma$. A non-empty subset A of $S$ is called a $\Gamma$-hypersubsemigroup of $S$ if $a_{1} \gamma a_{2} \subseteq A$ for every $a_{1}, a_{2} \in A$. A $\Gamma$-semihypergroup $S$ is called commutative if for all $\mathrm{x}, \mathrm{y} \in \mathrm{S}$ and $\gamma \in \Gamma$ we have $\mathrm{x} \gamma \mathrm{y}=\mathrm{y} \gamma \mathrm{x}$.

Definition 2.5 [8 ] Let $U$ be an universel set and $E$ be the set of parameters. $P(U)$ denote the power set of $U$. Let $A$ be a non empty subset of $E$ then the pair $(F, A)$ is called a soft set over $U$, where $F$ is a mapping given by $F: A \rightarrow P(U)$.

\section{Definition 2.6}

[18] Let $X$ be a non-empty set. A fuzzy subset $\mu$ of $X$ is a function from $X$ into the closed unit interval $[0,1]$. The set of all fuzzy subset of $X$ is called the fuzzy power set of $X$ and is denoted by $\mathrm{FP}(\mathrm{X})$. 


\section{Definition 2.7[4]}

A bipolar fuzzy set $A$ in a universe $U$ is an object having the form $\mathrm{A}=\left\{\left\langle\mathrm{x}, \mu_{\mathrm{A}}^{+}(\mathrm{x}), \mu_{\mathrm{A}}^{-}(\mathrm{x})\right\rangle: \mathrm{x} \in \mathrm{X}\right\}$, where $\mu_{\mathrm{A}}^{+}: \mathrm{X} \rightarrow[0,1]$ and $\mu_{\mathrm{A}}^{-}: \mathrm{X} \rightarrow$ $[-1,0]$. Here $\mu_{A}^{+}(x)$ represents the degree of satisfaction of an element $x$ to the property and $A=\left\{\left\langle x, \mu_{A}^{+}(x), \mu_{A}^{-}(x)\right\rangle: x \in X\right\}$ and $\mu_{\mathrm{A}}^{-}(\mathrm{x})$ represents the degree of satisfaction of $\mathrm{x}$ to some implict counter property of $A$. For the simplicity the symbol $\left\langle\mu_{A}^{+}, \mu_{A}^{-}\right\rangle$is used for the bipolar fuzzy set $A=\left\{\left\langle x, \mu_{A}^{+}(x), \mu_{A}^{-}(x)\right\rangle: x \in X\right\}$.

\section{Definition 2.8 [2]}

Let $\mathrm{U}$ be the universe set and $\mathrm{E}$ be the set of parameter. Let $\mathrm{A} \subseteq \mathrm{E}$ and $\mathrm{BF}^{\mathrm{U}}$ denotes the set of all bipolar fuzzy subsets of $\mathrm{U}$. Then a pair $(F, A)$ is called a bipolar fuzzy soft sets over $U$, where $F$ is a mapping given by $\mathrm{F}: \mathrm{A} \rightarrow \mathrm{BF}^{\mathrm{U}}$.

It is defined as $(\mathrm{F}, \mathrm{A})=\left\{\left\langle\mathrm{x}, \mu_{\mathrm{a}}^{+}(\mathrm{x}), \mu_{\mathrm{a}}^{-}(\mathrm{x})\right\rangle: \mathrm{x} \in\right.$ Uand $\left.\mathrm{a} \in \mathrm{A}\right\}$ For any

$$
\begin{gathered}
\mathrm{a} \in \mathrm{A}, \mathrm{F}(\mathrm{a})=\left\{\left\langle\mathrm{x}, \mu_{\mathrm{F}(\mathrm{a})}^{+}(\mathrm{x}), \mu_{\mathrm{F}(\mathrm{a})}^{-}(\mathrm{x})\right\rangle: \mathrm{x} \in \mathrm{U}\right\} \\
=\left\langle\mu_{\mathrm{F}(\mathrm{a})}^{+}(\mathrm{x}), \mu_{\mathrm{F}(\mathrm{a})}^{-}(\mathrm{x})\right\rangle .
\end{gathered}
$$

\section{Definition 2.9 [2]}

Let $(F, A)$ and $(G, B)$ be two bipolar fuzzy soft sets over a common universe $\mathrm{U}$, then $(\mathrm{F}, \mathrm{A})$ AND $(\mathrm{G}, \mathrm{B})$ denoted by $(F, A) \wedge(G, B)$ is defined as $(F, A) \wedge(G, B)=(H, C)$ where $\mathrm{C}=\mathrm{A} \times \mathrm{B}$ and $\mathrm{H}(\mathrm{a}, \mathrm{b})=\mathrm{F}(\mathrm{a}) \cap \mathrm{G}(\mathrm{b})$, for all $(\mathrm{a}, \mathrm{b}) \in \mathrm{A} \times \mathrm{B}$.

\section{Definition $2.101[2]$}

Let $(F, A)$ and $(G, B)$ be two bipolar fuzzy soft sets over a common universe $\mathrm{U}$, then $(\mathrm{F}, \mathrm{A})$ OR $(\mathrm{G}, \mathrm{B})$ denoted by $(\mathrm{F}, \mathrm{A}) \mathrm{V}$ $(\mathrm{G}, \mathrm{B})$ is defined as $(\mathrm{F}, \mathrm{A}) \vee(\mathrm{G}, \mathrm{B})=(\mathrm{H}, \mathrm{C})$ where $\mathrm{C}=\mathrm{A} \times \mathrm{B}$ and $H(a, b)=F(a) \cup G(b)$, for all $(a, b) \in A \times B$.

\section{Definition 2.11 [2]}

Let $(F, A)$ and $(G, B)$ be two bipolar fuzzy soft sets over a common universe $U$ then their extended union is a bipolar fuzzy soft set over $U$ denoted by $(F, A) \cup_{\varepsilon}(G, B)$ and is defined by $(\mathrm{F}, \mathrm{A}) \cup_{\varepsilon}(\mathrm{G}, \mathrm{B})=(\mathrm{H}, \mathrm{C})$ where $\mathrm{C}=\mathrm{A} \cup \mathrm{B}, \mathrm{H}: \mathrm{C} \rightarrow \mathrm{BF}^{\mathrm{U}}$ and

$$
H(c)=\left\{\begin{array}{l}
F(c) \text { if } c \in A-B \\
G(c) \text { if } c \in B-A \\
F(c) \cup G(c) \text { if } c \in A \cap B
\end{array}\right.
$$

\section{Definition $2.12[2]$}

Let $(F, A)$ and $(G, B)$ be two bipolar fuzzy soft sets over a common universe $U$ then their extended intersection is a bipolar fuzzy soft set over $U$ denoted by $(F, A) \cap_{\varepsilon}(G, B)$ and is defined by $(\mathrm{F}, \mathrm{A}) \mathrm{U}_{\varepsilon}(\mathrm{G}, \mathrm{B})=(\mathrm{H}, \mathrm{C})$ where $\mathrm{C}=\mathrm{A} \cup \mathrm{B}, \mathrm{H}: \mathrm{C} \rightarrow \mathrm{BF}^{\mathrm{U}}$ and

$$
H(c)=\left\{\begin{array}{l}
F(c) \text { if } c \in A-B \\
G(c) \text { if } c \in B-A \\
F(c) \cap G(c) \text { if } c \in A \cap B
\end{array}\right.
$$

\section{Definition 2.13[13 ]}

Let $(F, A)$ and $(G, B)$ be two bipolar fuzzy soft sets over a common universe $U$ such that $A \cap B \neq \phi$. The restricted union of $(F, A)$ and $(G, B)$ is defined to be a bipolar fuzzy soft set $(H, C)$ over $U$ where $C=A \cap B$ and $H(c)=F(c) \cup G(c)$, for all $c \in C$. This is denoted by $(H, C)=(F, A) \cup_{R}(G, B)$.

\section{Definition $2.14[11]$}

Let $(F, A)$ and $(G, B)$ be two bipolar fuzzy soft sets over a common universe $U$ such that $A \cap B \neq \phi$. The restricted intersection of $(F, A)$ and $(G, B)$ is defined to be a bipolar fuzzy soft set $(H, C)$ over $U$ where $C=A \cap B$ and $H(c)=F(c) \cap$ $\mathrm{G}(\mathrm{c})$, for all $\mathrm{c} \in \mathrm{C}$. This is denoted by $(\mathrm{H}, \mathrm{C})=(\mathrm{F}, \mathrm{A}) \cap_{\mathrm{R}}(\mathrm{G}, \mathrm{B})$.

\section{Definition 2.15 [9]}

Let $(F, A)$ be a bipolar fuzzy soft set over $U$ for each $t \in[0,1]$ and $s \in[-1,0]$ the set $(F, A)^{(t, s)}=\left(F^{(t, s)}, A\right)$ where $(F, A)_{a}^{(t, s)}=$ $\left\{\mathrm{x} \in \mathrm{U} \mid \mu_{\mathrm{F}(\mathrm{a})}^{\mathrm{P}}(\mathrm{x}) \geq \mathrm{t}, \mu_{\mathrm{F}(\mathrm{a})}^{\mathrm{N}}(\mathrm{x}) \leq \mathrm{s}\right\}$ for all $\mathrm{a} \in \mathrm{A}$.

\section{Definition 2.16[17]}

Let $\phi: \mathrm{H}_{1} \rightarrow \mathrm{H}_{2}$ and $\mathrm{h}: \mathrm{E}_{1} \rightarrow \mathrm{E}_{2}$ be two maps, $\mathrm{A} \subseteq \mathrm{E}_{1}$ and $\mathrm{B} \subseteq \mathrm{E}_{2}$, where $\mathrm{E}_{1}$ and $\mathrm{E}_{2}$ are sets of parameters viewed on $\mathrm{H}_{1}$ and $\mathrm{H}_{2}$, respectively. The pair $(\phi, h)$ is called a fuzzy soft map from $\mathrm{H}_{1}$ to $\mathrm{H}_{2}$. If $\phi$ is a hypergroup homomorphism, then $(\phi, h)$ is called a fuzzy soft homomorphism from $\mathrm{H}_{1}$ to $\mathrm{H}_{2}$.

\section{Definition 2.17 [3]}

Let (f, A) and (g, B) be two fuzzy soft sets over $\mathrm{H}_{1}$ and $\mathrm{H}_{2}$, respectively, and $(\phi, h)$ be a fuzzy soft function from $\mathrm{H}_{1}$ to $\mathrm{H}_{2}$ (i) The image of (f, A) under the soft function $(\phi, h)$ denoted by $(\phi, h)(f, A)$, is a fuzzy soft set over $\mathrm{H}_{2}$ defined by $(\phi, h)(f, A)=$ $(\phi(f), h(A))$, where for all $b \in h(A)$ and for all $y \in H_{2}$, then

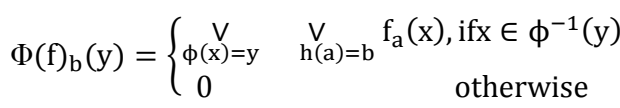

(ii) The inverse image of (g, B) under the fuzzy soft function $(\phi, h)$ denoted by $(\phi, h)^{-1}(\mathrm{~g}, \mathrm{~B})$, is a fuzzy soft set over B defined by $(\phi, h)^{-1}(g, B)=\left(\phi^{-1}(g), h^{-1}(A)\right)$, where for all $\mathrm{a} \in \mathrm{h}^{-1}(\mathrm{~A})$ and for all $\mathrm{x} \in \mathrm{H}_{1}, \phi^{-1}(\mathrm{~g})_{\mathrm{a}}(\mathrm{x})=\mathrm{g}_{\mathrm{h}(\mathrm{a})}(\phi(\mathrm{x}))$. If $\phi$ and $h$ is injective(surjective), then $(\phi, h)$ is said to be injective (surjective).

\section{Definition 2.18 [15]}

Let $(\phi, \psi)$ be a fuzzy soft $\Gamma$-function from X to Y. If $\phi$ is a homomorphism function from X to $\mathrm{Y}$, then $(\phi, \psi)$ is said to be fuzzy soft $\Gamma$-homomorphism. If $\phi$ is isomorphism function from $\mathrm{X}$ to $\mathrm{Y}$ and $\psi$ is one to one mapping from $\mathrm{N}$ to $\mathrm{M}$, then $(\phi, \psi)$ is said to be fuzzy soft $\Gamma$-isomorphism.

\section{Bipolar Fuzzy Soft $\Gamma$ - Hyper Ideals}

In this section, we introduce the notion of bipolar fuzzy soft gamma hyperideals in gamma semigroups and discuss some of its properties $\mathrm{S}$ denotes the $\Gamma$ - hyper semigroup.

\section{Definition 3.1}

A bipolar fuzzy soft set $(F, A)$ over a $\Gamma$-hypersemigroups $S$ is called a bipolar fuzzy soft $\Gamma$-subhypersemigroup over $\mathrm{S}$ if

(i) $\inf _{\mathrm{x} \in \mathrm{y} \gamma \mathrm{z}} \mu_{\mathrm{F}(\mathrm{a})}^{+}(\mathrm{x}) \geq \min \left\{\mu_{\mathrm{F}(\mathrm{a})}^{+}(\mathrm{y}), \mu_{\mathrm{F}(\mathrm{a})}^{+}(\mathrm{z})\right\}$

(ii) $\sup _{\mathrm{x} \in \mathrm{y} \gamma \mathrm{z}} \mu_{\mathrm{F}(\mathrm{a})}^{-}(\mathrm{x}) \leq \max \left\{\mu_{\mathrm{F}(\mathrm{a})}^{-}(\mathrm{y}), \mu_{\mathrm{F}(\mathrm{a})}^{-}(\mathrm{z})\right\}$ for all $\mathrm{x}, \mathrm{y}, \mathrm{z} \in \mathrm{S}$, $\gamma \in \Gamma$ and $a \in A$.

\section{Definition 3.2}

A bipolar fuzzy soft set $(F, A)$ over a $\Gamma$ - hypersemigroups $S$ is called a bipolar fuzzy soft left $\Gamma$-hyperideal over $S$ if

(i) $\inf _{\mathrm{x} \in \mathrm{y} \gamma \mathrm{z}} \mu_{\mathrm{F}(\mathrm{a})}^{+}(\mathrm{x}) \geq \mu_{\mathrm{F}(\mathrm{a})}^{+}(\mathrm{z})$

(ii) $\sup _{\mathrm{x} \in \mathrm{y} \gamma \mathrm{z}} \mu_{\mathrm{F}(\mathrm{a})}^{-}(\mathrm{x}) \leq \mu_{\mathrm{F}(\mathrm{a})}^{-}(\mathrm{z})$ for all $\mathrm{x}, \mathrm{y}, \mathrm{z} \in \mathrm{S}, \gamma \in \Gamma$ and $\mathrm{a} \in \mathrm{A}$.

Definition 3.3A bipolar fuzzy soft set $(F, A)$ over a $\Gamma$ hypersemigroups $\mathrm{S}$ is called a bipolar fuzzy soft right $\Gamma$-hyperideal over $\mathrm{S}$ if

(i) $\inf _{\mathrm{x} \in \mathrm{y} \gamma \mathrm{z}} \mu_{\mathrm{F}(\mathrm{a})}^{+}(\mathrm{x}) \geq \mu_{\mathrm{F}(\mathrm{a})}^{+}(\mathrm{y})$ (ii) $\sup _{\mathrm{x} \in \mathrm{y} \gamma \mathrm{z}} \mu_{\mathrm{F}(\mathrm{a})}^{-}(\mathrm{x}) \leq \mu_{\mathrm{F}(\mathrm{a})}^{-}(\mathrm{y})$ for all $\mathrm{x}, \mathrm{y}, \mathrm{z} \in \mathrm{S}, \gamma \in \Gamma$ and $\mathrm{a} \in \mathrm{A}$.

\section{Definition 3.4}

A bipolar fuzzy soft set $(F, A)$ over a $\Gamma$-hypersemigroups $S$ is called a bipolar fuzzy soft $\Gamma$-hyperideal of $S$ if

(i) $\inf _{\mathrm{x} \in \mathrm{y} \gamma \mathrm{z}} \mu_{\mathrm{F}(\mathrm{a})}^{+}(\mathrm{x}) \geq \max \left\{\mu_{\mathrm{F}(\mathrm{a})}^{+}(\mathrm{y}), \mu_{\mathrm{F}(\mathrm{a})}^{+}(\mathrm{z})\right\}$ 
(ii) $\sup _{\mathrm{x} \in \mathrm{y} \gamma \mathrm{z}} \mu_{\mathrm{F}(\mathrm{a})}^{-}(\mathrm{x}) \leq \min \left\{\mu_{\mathrm{F}(\mathrm{a})}^{-}(\mathrm{y}), \mu_{\mathrm{F}(\mathrm{a})}^{-}(\mathrm{z})\right\}$ for all $\mathrm{x}, \mathrm{y}, \mathrm{z} \in \mathrm{S}$, $\gamma \in \Gamma$ and $a \in A$.

\section{Definition 3.5}

A bipolar fuzzy soft set $(F, A)$ over a $\Gamma$-hypersemigroups $S$ is called a bipolar fuzzy soft $\Gamma$ - hyperbi-ideal over $S$ if

(i) $\inf _{\mathrm{p} \in \mathrm{x} \propto \mathrm{y} \beta \mathrm{z}} \mu_{\mathrm{F}(\mathrm{a})}^{+}(\mathrm{p}) \geq \min \left\{\mu_{\mathrm{F}(\mathrm{a})}^{+}(\mathrm{x}), \mu_{\mathrm{F}(\mathrm{a})}^{+}(\mathrm{z})\right\}$

(ii) $\sup _{\mathrm{p} \in \mathrm{x} \propto y \beta z} \mu_{\mathrm{F}(\mathrm{a})}^{-}(\mathrm{p}) \leq \max \left\{\mu_{\mathrm{F}(\mathrm{a})}^{-}(\mathrm{x}), \mu_{\mathrm{F}(\mathrm{a})}^{-}(\mathrm{z})\right\}$ for all $\mathrm{x}, \mathrm{y}, \mathrm{z} \in \mathrm{S}$, $\alpha, \beta \in \Gamma$ and $\mathrm{a} \in \mathrm{A}$.

\section{Definition 3.6}

A bipolar fuzzy soft set $(F, A)$ over a $\Gamma$-hypersemigroups $S$ is called a bipolar fuzzy soft $\Gamma$-hyperinterior ideal over $S$ if

(i) $\inf _{\mathrm{p} \in \mathrm{x} \propto \mathrm{y} \beta \mathrm{z}} \mu_{\mathrm{F}(\mathrm{a})}^{+}(\mathrm{p}) \geq \mu_{\mathrm{F}(\mathrm{a})}^{+}(\mathrm{y})$

(ii) $\sup _{\mathrm{p} \in \mathrm{x} \alpha \mathrm{y} \beta \mathrm{z}} \mu_{\mathrm{F}(\mathrm{a})}^{-}(\mathrm{p}) \leq \mu_{\mathrm{F}(\mathrm{a})}^{-}(\mathrm{y})$ for all $\mathrm{x}, \mathrm{y}, \mathrm{z} \in \mathrm{S}, \alpha, \beta \in \Gamma$ and $a \in A$.

\section{Theorem 3.7}

Let $(F, A)$ and $(G, B)$ be two bipolar fuzzy soft

$\Gamma$-hypersubsemigroups over $S$, then $(F, A) \wedge(G, B)$ and $(F, A) \vee$

$(G, B)$ are bipolar fuzzy soft $\Gamma$ - hypersubsemigroup of $S$.

Proof. Let $(F, A)$ and $(G, B)$ be two bipolar fuzzy soft $\Gamma$-hypersubsemigroups over $S$ defined as $(F, A) \wedge(G, B)$ where $\mathrm{C}=\mathrm{A} \times \mathrm{B}$ and $\mathrm{H}(\mathrm{a}, \mathrm{b})=\mathrm{F}(\mathrm{a}) \cap \mathrm{G}(\mathrm{b})$, for all $(\mathrm{a}, \mathrm{b}) \in \mathrm{C}=\mathrm{A} \times$ $\mathrm{B}, \mathrm{x}, \mathrm{y}, \mathrm{z} \in \mathrm{S} \gamma \in \Gamma$

$$
\begin{aligned}
& \inf _{\mathrm{z} \in \mathrm{x} \gamma \mathrm{y}}\left\{\mu_{\mathrm{H}(\mathrm{a}, \mathrm{b})}^{+}(\mathrm{z})\right\}=\inf _{\mathrm{z} \in \mathrm{x} \gamma \mathrm{y}}\left\{\min \left\{\mu_{\mathrm{F}(\mathrm{a})}^{+}(\mathrm{z}), \mu_{\mathrm{G}(\mathrm{b})}^{+}(\mathrm{z})\right\}\right\} \\
& =\min \left\{\inf _{\mathrm{z} \in \mathrm{x} \gamma \mathrm{y}} \mu_{\mathrm{F}(\mathrm{a})}^{+}(\mathrm{z}), \inf _{\mathrm{z} \in \mathrm{x} \gamma \mathrm{y}} \mu_{\mathrm{G}(\mathrm{b})}^{+}(\mathrm{z})\right\} \\
& \geq \min \left\{\min \left\{\mu_{\mathrm{F}(\mathrm{a})}^{+}(\mathrm{x}), \mu_{\mathrm{F}(\mathrm{a})}^{+}(\mathrm{y})\right\}, \min \left\{\mu_{\mathrm{G}(\mathrm{b})}^{+}(\mathrm{x}), \mu_{\mathrm{G}(\mathrm{b})}^{+}(\mathrm{y})\right\}\right\} \\
& =\min \left\{\left\{\min \left\{\mu_{\mathrm{F}(\mathrm{a})}^{+}(\mathrm{x}), \mu_{\mathrm{G}(\mathrm{b})}^{+}(\mathrm{x})\right\}, \min \left\{\mu_{\mathrm{F}(\mathrm{a})}^{+}(\mathrm{q}), \mu_{\mathrm{G}(\mathrm{b})}^{+}(\mathrm{q})\right\}\right\}\right. \\
& =\min \left\{\left(\mu_{\mathrm{F}(\mathrm{a})}^{+} \cap \mu_{\mathrm{G}(\mathrm{b})}^{+}\right)(\mathrm{x}),\left(\mu_{\mathrm{F}(\mathrm{a})}^{+} \cap \mu_{\mathrm{G}(\mathrm{b})}^{+}\right)(\mathrm{y})\right\} \\
& =\min \left\{\mu_{\mathrm{H}(\mathrm{a}, \mathrm{b})}^{+}(\mathrm{x}), \mu_{\mathrm{H}(\mathrm{a}, \mathrm{b})}^{+}(\mathrm{y})\right\} \text {. } \\
& \sup _{\mathrm{z} \in \mathrm{x} \gamma \mathrm{y}}\left\{\mu_{\mathrm{H}(\mathrm{a}, \mathrm{b})}^{-}(\mathrm{z})\right\}=\sup _{\mathrm{z} \in \mathrm{x} \gamma \mathrm{y}}\left\{\max \left\{\mu_{\mathrm{F}(\mathrm{a})}^{-}(\mathrm{z}), \mu_{\mathrm{G}(\mathrm{b})}^{-}(\mathrm{z})\right\}\right\} \\
& =\max \left\{\sup _{\mathrm{z} \in \mathrm{x} \gamma \mathrm{y}} \mu_{\mathrm{F}(\mathrm{a})}^{+}(\mathrm{z}), \inf _{\mathrm{z} \in \mathrm{x} \gamma \mathrm{y}} \mu_{\mathrm{G}(\mathrm{b})}^{+}(\mathrm{z})\right\} \\
& \geq \max \left\{\max \left\{\mu_{\mathrm{F}(\mathrm{a})}^{-}(\mathrm{x}), \mu_{\mathrm{F}(\mathrm{a})}^{-}(\mathrm{y})\right\}, \min \left\{\mu_{\mathrm{G}(\mathrm{b})}^{-}(\mathrm{x}), \mu_{\mathrm{G}(\mathrm{b})}^{-}(\mathrm{y})\right\}\right\} \\
& =\max \left\{\left\{\max \left\{\mu_{\mathrm{F}(\mathrm{a})}^{+}(\mathrm{x}), \mu_{\mathrm{G}(\mathrm{b})}^{+}(\mathrm{x})\right\}, \max \left\{\mu_{\mathrm{F}(\mathrm{a})}^{-}(\mathrm{y}), \mu_{\mathrm{G}(\mathrm{b})}^{-}(\mathrm{y})\right\}\right\}\right. \\
& =\max \left\{\left(\mu_{\mathrm{F}(\mathrm{a})}^{-} \cup \mu_{\mathrm{G}(\mathrm{b})}^{-}\right)(\mathrm{x}),\left(\mu_{\mathrm{F}(\mathrm{a})}^{-} \cup \mu_{\mathrm{G}(\mathrm{b})}^{-}\right)(\mathrm{y})\right\} \\
& =\max \left\{\mu_{\mathrm{H}(\mathrm{a}, \mathrm{b})}^{-}(\mathrm{x}), \mu_{\mathrm{H}(\mathrm{a}, \mathrm{b})}^{-}(\mathrm{y})\right\} \text {. }
\end{aligned}
$$

Hence $(\mathrm{F}, \mathrm{A}) \wedge(\mathrm{G}, \mathrm{B})$ is a bipolar fuzzy soft $\Gamma$-hypersubsemigroup over $S$.Similarly it can be shown that $(F, A) \vee(G, B)$ are bipolar fuzzy soft $\Gamma$-hypersub semigroup over $\mathrm{S}$.

\section{Theorem 3.8}

Let $(F, A)$ and $(G, B)$ be two bipolar fuzzy soft $\Gamma$-hyperleft (resp.right) ideals over $S$, then $(F, A) \wedge(G, B)$ and $(F, A) \vee(G, B)$ are bipolar fuzzy soft $\Gamma$-hyperleft (resp.right) ideals of $S$.

Proof. Let $(F, A)$ and $(G, B)$ be two bipolar fuzzy soft $\Gamma$-hyperleftideals over $S$ defined as $(F, A) \wedge(G, B)$ where $\mathrm{C}=\mathrm{A} \times \mathrm{B}$ and $\mathrm{H}(\mathrm{a}, \mathrm{b})=\mathrm{F}(\mathrm{a}) \cap \mathrm{G}(\mathrm{b})$, for all $(\mathrm{a}, \mathrm{b}) \in \mathrm{C}=\mathrm{A} \times$ $\mathrm{B}, \mathrm{x}, \mathrm{y}, \mathrm{z} \in \mathrm{S}$ and $\gamma \in \Gamma$.

$$
\begin{aligned}
& \inf _{\mathrm{z} \in \mathrm{x} \gamma \mathrm{y}}\left\{\mu_{\mathrm{H}(\mathrm{a}, \mathrm{b})}^{+}(\mathrm{z})\right\}=\inf _{\mathrm{z} \in \mathrm{x} \gamma \mathrm{y}}\left\{\min \left\{\mu_{\mathrm{F}(\mathrm{a})}^{+}(\mathrm{z}), \mu_{\mathrm{G}(\mathrm{b})}^{+}(\mathrm{z})\right\}\right\} \\
& =\min \left\{\inf _{\mathrm{z} \in \mathrm{x} \gamma \mathrm{y}} \mu_{\mathrm{F}(\mathrm{a})}^{+}(\mathrm{z}), \inf _{\mathrm{z} \in \mathrm{x} \gamma \mathrm{y}} \mu_{\mathrm{G}(\mathrm{b})}^{+}(\mathrm{z})\right\} \\
& =\min \left\{\mu_{\mathrm{F}(\mathrm{a})}^{+}(\mathrm{y}), \mu_{\mathrm{G}(\mathrm{b})}^{+}(\mathrm{y})\right\} \\
& \left.=\mu_{\mathrm{H}(\mathrm{a}, \mathrm{b})}^{+}(\mathrm{y})\right\} . \\
& \quad \text { and }
\end{aligned}
$$

$$
\begin{aligned}
& \sup _{\mathrm{z} \in \mathrm{x} \gamma \mathrm{y}}\left\{\mu_{\mathrm{H}(\mathrm{a}, \mathrm{b})}^{-}(\mathrm{z})\right\}=\underset{\mathrm{z} \in \mathrm{x} \gamma \mathrm{y}}{\sup }\left\{\max \left\{\mu_{\mathrm{F}(\mathrm{a})}^{-}(\mathrm{z}), \mu_{\mathrm{G}(\mathrm{b})}^{-}(\mathrm{z})\right\}\right\} \\
& \quad=\max \left\{\sup _{\mathrm{z} \in \mathrm{x} \gamma \mathrm{y}} \mu_{\mathrm{F}(\mathrm{a})}^{-}(\mathrm{z}), \inf _{\mathrm{z} \in \mathrm{x} \gamma \mathrm{y}} \mu_{\mathrm{G}(\mathrm{b})}^{-}(\mathrm{z})\right\} \\
& \leq \max \left\{\mu_{\mathrm{F}(\mathrm{a})}^{-}(\mathrm{y}), \mu_{\mathrm{G}(\mathrm{b})}^{-}(\mathrm{y})\right\} \\
& =\mu_{\mathrm{H}(\mathrm{a}, \mathrm{b})}^{-}(\mathrm{y})
\end{aligned}
$$

Hence $(F, A) \wedge(G, B)$ are bipolar fuzzy soft $\Gamma$-left (resp.right) hyperideals over $\mathrm{S}$.

Similar proof shows that $(F, A) \vee(G, B)$ is a bipolar fuzzy soft $\Gamma$-left (resp.right) hyperideals over $S$.

\section{Theorem 3.9}

Let $(F, A)$ and $(G, B)$ be two bipolar fuzzy soft $\Gamma$-hyperbi-ideals over $S$, then $(F, A) \wedge(G, B)$ and $(F, A) \vee(G, B)$ are bipolar fuzzy soft $\Gamma$-hyperbi-ideals of $S$.

Proof. Let $(F, A)$ and $(G, B)$ be two bipolar fuzzy soft $\Gamma$-hypersemigroups over $S$ defined as $(F, A) \wedge(G, B)$ where $\mathrm{C}=\mathrm{A} \times \mathrm{B}, \mathrm{H}(\mathrm{a}, \mathrm{b})=\mathrm{F}(\mathrm{a}) \cap \mathrm{G}(\mathrm{b})$, for all $(\mathrm{a}, \mathrm{b}) \in \mathrm{C}=\mathrm{A} \times \mathrm{B}$, $\mathrm{x}, \mathrm{y}, \mathrm{z} \in \mathrm{S}$ and $\gamma \in \Gamma$.

$$
\begin{aligned}
& \inf _{\mathrm{z} \in \mathrm{x} \alpha \mathrm{y} \beta \mathrm{z}}\left\{\mu_{\mathrm{H}(\mathrm{a}, \mathrm{b})}^{+}(\mathrm{z})\right\}=\inf _{\mathrm{z} \in \mathrm{x} \gamma \mathrm{y}}\left\{\min \left\{\mu_{\mathrm{F}(\mathrm{a})}^{+}(\mathrm{z}), \mu_{\mathrm{G}(\mathrm{b})}^{+}(\mathrm{z})\right\}\right\} \\
& =\min \left\{\inf _{\mathrm{z} \in \mathrm{x} \gamma \mathrm{y}} \mu_{\mathrm{F}(\mathrm{a})}^{+}(\mathrm{z}), \inf _{\mathrm{z} \in \mathrm{x} \gamma \mathrm{y}} \mu_{\mathrm{G}(\mathrm{b})}^{+}(\mathrm{z})\right\} \\
& \geq \min \left\{\min \left\{\mu_{\mathrm{F}(\mathrm{a})}^{+}(\mathrm{x}), \mu_{\mathrm{F}(\mathrm{a})}^{+}(\mathrm{z})\right\}, \min \left\{\mu_{\mathrm{G}(\mathrm{b})}^{+}(\mathrm{x}), \mu_{\mathrm{G}(\mathrm{b})}^{+}(\mathrm{z})\right\}\right\} \\
& =\min \left\{\left\{\min \left\{\mu_{\mathrm{F}(\mathrm{a})}^{+}(\mathrm{x}), \mu_{\mathrm{G}(\mathrm{b})}^{+}(\mathrm{x})\right\}, \min \left\{\mu_{\mathrm{F}(\mathrm{a})}^{+}(\mathrm{z}), \mu_{\mathrm{G}(\mathrm{b})}^{+}(\mathrm{z})\right\}\right\}\right. \\
& =\min \left\{\left(\mu_{\mathrm{F}(\mathrm{a})}^{+} \cap \mu_{\mathrm{G}(\mathrm{b})}^{+}\right)(\mathrm{x}),\left(\mu_{\mathrm{F}(\mathrm{a})}^{+} \cap \mu_{\mathrm{G}(\mathrm{b})}^{+}\right)(\mathrm{z})\right\} \\
& =\min \left\{\mu_{\mathrm{H}(\mathrm{a}, \mathrm{b})}^{+}(\mathrm{x}), \mu_{\mathrm{H}(\mathrm{a}, \mathrm{b})}^{+}(\mathrm{z})\right\} . \\
& \sup _{\mathrm{z} \in \mathrm{x} \alpha \mathrm{y} \beta \mathrm{z}}\left\{\mu_{\mathrm{H}(\mathrm{a}, \mathrm{b})}^{-}(\mathrm{z})\right\}=\sup _{\mathrm{z} \in \mathrm{x} \gamma \mathrm{y}}\left\{\max \left\{\mu_{\mathrm{F}(\mathrm{a})}^{-}(\mathrm{z}), \mu_{\mathrm{G}(\mathrm{b})}^{-}(\mathrm{z})\right\}\right\} \\
& =\max \left\{\sup _{\mathrm{z} \in \mathrm{x} \gamma \mathrm{y}}^{+} \mu_{\mathrm{F}(\mathrm{a})}^{+}(\mathrm{z}), \inf _{\mathrm{z} \in \mathrm{x} \gamma \mathrm{y}} \mu_{\mathrm{G}(\mathrm{b})}^{+}(\mathrm{z})\right\} \\
& \leq \max \left\{\max \left\{\mu_{\mathrm{F}(\mathrm{a})}^{-}(\mathrm{x}), \mu_{\mathrm{F}(\mathrm{a})}^{-}(\mathrm{z})\right\}, \min \left\{\mu_{\mathrm{G}(\mathrm{b})}^{-}(\mathrm{x}), \mu_{\mathrm{G}(\mathrm{b})}^{-}(\mathrm{z})\right\}\right\} \\
& =\max \left\{\left\{\max \left\{\mu_{\mathrm{F}(\mathrm{a})}^{-}(\mathrm{x}), \mu_{\mathrm{G}(\mathrm{b})}^{-}(\mathrm{x})\right\}, \max \left\{\mu_{\mathrm{F}(\mathrm{a})}^{-}(\mathrm{z}), \mu_{\mathrm{G}(\mathrm{b})}^{-}(\mathrm{z})\right\}\right\}\right. \\
& =\max \left\{\left(\mu_{\mathrm{F}(\mathrm{a})}^{-} \cup \mu_{\mathrm{G}(\mathrm{b})}^{-}\right)(\mathrm{x}),\left(\mu_{\mathrm{F}(\mathrm{a})}^{-} \cup \mu_{\mathrm{G}(\mathrm{b})}^{-}\right)(\mathrm{z})\right\} \\
& =\max \left\{\mu_{\mathrm{H}(\mathrm{a}, \mathrm{b})}^{-}(\mathrm{x}), \mu_{\mathrm{H}(\mathrm{a}, \mathrm{b})}^{-}(\mathrm{z})\right\} .
\end{aligned}
$$

Hence $(F, A) \wedge(G, B)$ is a bipolar fuzzy soft $\Gamma$-hyperbi-ideal over S.

It can be similarly proved that $(F, A) \vee(G, B)$ is a bipolar fuzzy soft $\Gamma$-hyperbi-ideal over S.

\section{Theorem 3.10}

Let $(F, A)$ and $(G, B)$ be two bipolar fuzzy soft $\Gamma$-hypersubsemigroups over $S$, then $(F, A) \cap_{\varepsilon}(G, B)$ is a bipolar fuzzy soft $\Gamma$-hypersubsemigroups of $S$.

Proof. Let $(F, A)$ and $(G, B)$ be two bipolar fuzzy soft $\Gamma$-hypersubsemigroups over $S$ as defined

$$
(\mathrm{F}, \mathrm{A}) \cap_{\varepsilon}(\mathrm{G}, \mathrm{B})=(\mathrm{H}, \mathrm{C}) \text { where } \mathrm{C}=\mathrm{A} \cup \mathrm{B}
$$

$$
H(c)=\left\{\begin{array}{l}
F(c) \text { if } c \in A \backslash B \\
G(c) \text { if } c \in B \backslash A \\
F(c) \cap G(c) \text { if } c \in A \cap B
\end{array}\right.
$$

Case(i) $c \in A \backslash B$ and $\gamma \in \Gamma$

$$
\begin{aligned}
& \inf _{\mathrm{z} \in \mathrm{x} \gamma \mathrm{y}}\left\{\mu_{\mathrm{H}(\mathrm{c})}^{+}(\mathrm{z})\right\}=\inf _{\mathrm{z} \in \mathrm{x} \gamma \mathrm{y}} \mu_{\mathrm{F}(\mathrm{c})}^{+}(\mathrm{z}) \\
& \quad \geq \min \left\{\mu_{\mathrm{F}(\mathrm{c})}^{+}(\mathrm{x}), \mu_{\mathrm{F}(\mathrm{c})}^{+}(\mathrm{y})\right\} \\
& \quad=\min \left\{\mu_{\mathrm{H}(\mathrm{c})}^{+}(\mathrm{x}), \mu_{\mathrm{H}(\mathrm{c})}^{+}(\mathrm{y})\right\} \\
& \text { and } \\
& \sup _{\mathrm{z} \in \mathrm{x} \gamma \mathrm{y}}\left\{\mu_{\mathrm{H}(\mathrm{c})}^{-}(\mathrm{z})\right\}=\sup _{\mathrm{z} \in \mathrm{x} \gamma \mathrm{y}} \mu_{\mathrm{F}(\mathrm{c})}^{-}(\mathrm{z}) \\
& \quad \leq \max \left\{\mu_{\mathrm{F}(\mathrm{c})}^{-}(\mathrm{x}), \mu_{\mathrm{F}(\mathrm{c})}^{-}(\mathrm{y})\right\} \\
& =\max \left\{\mu_{\mathrm{H}(\mathrm{c})}^{-}(\mathrm{x}), \mu_{\mathrm{H}(\mathrm{c})}^{-}(\mathrm{y})\right\}
\end{aligned}
$$

Case(ii) $c \in B \backslash A$ and $\gamma \in \Gamma$. 


$$
\begin{aligned}
& \inf _{\mathrm{z} \in \mathrm{x} \gamma \mathrm{y}}\left\{\mu_{\mathrm{H}(\mathrm{c})}^{+}(\mathrm{z})\right\}=\inf _{\mathrm{z} \in \mathrm{x} \gamma \mathrm{y}} \mu_{\mathrm{G}(\mathrm{c})}^{+}(\mathrm{z}) \\
& \quad \geq \min \left\{\mu_{\mathrm{G}(\mathrm{c})}^{+}(\mathrm{x}), \mu_{\mathrm{G}(\mathrm{c})}^{+}(\mathrm{y})\right\} \\
& \quad=\min \left\{\mu_{\mathrm{H}(\mathrm{c})}^{+}(\mathrm{x}), \mu_{\mathrm{H}(\mathrm{c})}^{+}(\mathrm{y})\right\} \\
& \text { and } \\
& \sup _{\mathrm{z} \in \mathrm{x} \gamma \mathrm{y}}\left\{\mu_{\mathrm{H}(\mathrm{c})}^{-}(\mathrm{z})\right\}=\sup _{\mathrm{z} \in \mathrm{x} \gamma \mathrm{y}} \mu_{\mathrm{G}(\mathrm{c})}^{-}(\mathrm{z}) \\
& \quad \leq \max \left\{\mu_{\mathrm{G}(\mathrm{c})}^{-}(\mathrm{x}), \mu_{\mathrm{G}(\mathrm{c})}^{-}(\mathrm{y})\right\} \\
& =\max \left\{\mu_{\mathrm{H}(\mathrm{c})}^{-}(\mathrm{x}), \mu_{\mathrm{H}(\mathrm{c})}^{-}(\mathrm{y})\right\}
\end{aligned}
$$

Case (iii) $\mathrm{C} \in \mathrm{A} \cap \mathrm{B}$ and $\gamma \in \Gamma$ then $\mathrm{H}(\mathrm{c})=\mathrm{F}$ (c) $\cap \mathrm{G}$ (c) then by theorem 3.7,

$$
\inf _{\mathrm{z} \in \mathrm{x} \gamma \mathrm{y}}\left\{\mu_{\mathrm{H}(\mathrm{c})}^{+}(\mathrm{z})\right\} \geq \inf _{\mathrm{z} \in \mathrm{x} \gamma \mathrm{y}}\left\{\mu_{\mathrm{H}(\mathrm{c})}^{+}(\mathrm{x}), \mu_{\mathrm{H}(\mathrm{c})}^{+}(\mathrm{y})\right\}
$$$$
=\min \left\{\mu_{\mathrm{H}(\mathrm{c})}^{+}(\mathrm{x}), \mu_{\mathrm{H}(\mathrm{c})}^{+}(\mathrm{y})\right\} \text {, }
$$

and

$\sup _{\mathrm{z} \in \mathrm{x} \gamma \mathrm{y}}\left\{\mu_{\mathrm{H}(\mathrm{c})}^{-}(\mathrm{z})\right\} \leq \sup _{\mathrm{z} \in \mathrm{x} \gamma \mathrm{y}}\left\{\mu_{\mathrm{H}(\mathrm{c})}^{-}(\mathrm{x}), \mu_{\mathrm{H}(\mathrm{c})}^{-}(\mathrm{y})\right\}$

$=\max \gamma\left\{\mu_{\mathrm{H}(\mathrm{c})}^{-}(\mathrm{x}), \mu_{\mathrm{H}(\mathrm{c})}^{-}(\mathrm{y})\right\}$.

Hence $(F, A) \cap_{\varepsilon}(G, B)$ is a bipolar fuzzy soft

$\Gamma$-hypersubsemigroup over $\mathrm{S}$.

\section{Theorem3.11}

Let $(F, A)$ and $(G, B)$ be two bipolar fuzzy soft

$\Gamma$-hypersubsemigroup over $S$, then $(F, A) U_{\varepsilon}(G, B)$ is a bipolar fuzzy soft $\Gamma$-hypersubsemigroup of $S$.

Proof. Straight forward.

\section{Theorem 3.12}

Let $(F, A)$ and $(G, B)$ be two bipolar fuzzy soft

$\Gamma$-hyperbi(interior) ideal over $S$, then $(F, A) \cap_{\varepsilon}(G, B)$ is a bipolar fuzzy soft $\Gamma$-hyperbi(interior) ideal of $S$.

Proof. Straight forward.

\section{Theorem 3.13}

Let $(F, A)$ and $(G, B)$ be two bipolar fuzzy soft- $\Gamma$-hyper bi(interior) ideal over $S$, then $(F, A) \cup_{\varepsilon}(G, B)$ is a bipolar fuzzy soft $\Gamma$ - hyperbi(interior) ideal of $S$.

Proof. Straight forward.

\section{Theorem 3.14}

Let $(F, A)$ and $(G, B)$ be two bipolar fuzzy soft $\Gamma$ hypersubsemigroup over $S$, then $(F, A) \cap_{R}(G, B)$ is a bipolar fuzzy soft $\Gamma$-hypersubsemigroup of $S$.

Proof. Let $(F, A)$ and $(G, B)$ be two bipolar fuzzy soft $\Gamma$ hypersubsemigroup over $S$, then $(F, A) \cap_{R}(G, B)=(H, C)$ where $\mathrm{C}=\mathrm{A} \cap \mathrm{B}$ and $\mathrm{H}(\mathrm{c})=\mathrm{F}(\mathrm{c}) \cap \mathrm{G}(\mathrm{c})$ for all $\mathrm{c} \in \mathrm{C}$.

$$
\begin{aligned}
& \inf _{\mathrm{z} \in \mathrm{x} \gamma \mathrm{y}} \mu_{\mathrm{H}(\mathrm{c})}^{+}(\mathrm{z})=\inf _{\mathrm{z} \in \mathrm{x} \gamma \mathrm{y}}\left\{\min \left\{\mu_{\mathrm{F}(\mathrm{c})}^{+}(\mathrm{z}), \mu_{\mathrm{G}(\mathrm{c})}^{+}(\mathrm{z})\right\}\right\} \\
& =\min \left\{\inf _{\mathrm{z} \in \mathrm{x} \gamma \mathrm{y}} \mu_{\mathrm{F}(\mathrm{c})}^{+}(\mathrm{z}), \inf _{\mathrm{z} \in \mathrm{x} \gamma \mathrm{y}} \mu_{\mathrm{G}(\mathrm{c})}^{+}(\mathrm{z})\right\} \\
& \geq \min \left\{\min \left\{\mu_{\mathrm{F}(\mathrm{c})}^{+}(\mathrm{x}), \mu_{\mathrm{F}(\mathrm{c})}^{+}(\mathrm{y})\right\}, \min \left\{\mu_{\mathrm{G}(\mathrm{c})}^{+}(\mathrm{x}), \mu_{\mathrm{G}(\mathrm{c})}^{+}(\mathrm{y})\right\}\right\} \\
& =\min \left\{\min \left\{\mu_{\mathrm{F}(\mathrm{c})}^{+}(\mathrm{x}), \mu_{\mathrm{G}(\mathrm{c})}^{+}(\mathrm{x})\right\}, \min \left\{\mu_{\mathrm{F}(\mathrm{c})}^{+}(\mathrm{x}), \mu_{\mathrm{G}(\mathrm{c})}^{+}(\mathrm{x})\right\}\right\} \\
& =\min \left\{\left(\mu_{\mathrm{F}(\mathrm{c})}^{+} \cap \mu_{\mathrm{G}(\mathrm{c})}^{+}\right)(\mathrm{x}),\left(\mu_{\mathrm{F}(\mathrm{c})}^{+} \cap \mu_{\mathrm{G}(\mathrm{c})}^{+}\right)(\mathrm{y})\right\} \\
& =\min \left\{\mu_{\mathrm{H}(\mathrm{c})}^{+}(\mathrm{x}), \mu_{\mathrm{H}(\mathrm{c})}^{+}(\mathrm{y})\right\} . \\
& \quad \operatorname{sip}_{\mathrm{z} \in \mathrm{x} \gamma \mathrm{y}} \mu_{\mathrm{H}(\mathrm{c})}^{-}(\mathrm{z})=\sup _{\mathrm{z} \in \mathrm{x} \gamma \mathrm{y}}\left\{\max \left\{\mu_{\mathrm{F}(\mathrm{c})}^{-}(\mathrm{z}), \mu_{\mathrm{G}(\mathrm{c})}^{-}(\mathrm{z})\right\}\right\} \\
& =\max \left\{\sup _{\mathrm{z} \in \mathrm{x} \gamma \mathrm{y}} \mu_{\mathrm{F}(\mathrm{c})}(\mathrm{z}), \sup _{\mathrm{z} \in \mathrm{x} \gamma \mathrm{y}}^{-} \mu_{\mathrm{G}(\mathrm{c})}^{-}(\mathrm{z})\right\} \\
& \leq \max \left\{\max \left\{\mu_{\mathrm{F}(\mathrm{c})}^{-}(\mathrm{x}), \mu_{\mathrm{F}(\mathrm{c})}^{-}(\mathrm{y})\right\}, \max \left\{\mu_{\mathrm{G}(\mathrm{c})}^{-}(\mathrm{x}), \mu_{\mathrm{G}(\mathrm{c})}^{-}(\mathrm{y})\right\}\right\} \\
& =\max \left\{\max \left\{\mu_{\mathrm{F}(\mathrm{c})}^{-}(\mathrm{x}), \mu_{\mathrm{G}(\mathrm{c})}^{-}(\mathrm{x})\right\}, \max \left\{\mu_{\mathrm{F}(\mathrm{c})}^{-}(\mathrm{y}), \mu_{\mathrm{G}(\mathrm{c})}^{-}(\mathrm{y})\right\}\right\} \\
& =\max \left\{\left(\mu_{\mathrm{F}(\mathrm{c})}^{-} \cap \mu_{\mathrm{G}(\mathrm{c})}^{-}\right)(\mathrm{x}),\left(\mu_{\mathrm{F}(\mathrm{c})}^{-} \cap \mu_{\mathrm{G}(\mathrm{c})}^{-}(\mathrm{y})\right\}\right. \\
& =\max \left\{\mu_{\mathrm{H}(\mathrm{c})}^{-}(\mathrm{x}), \mu_{\mathrm{H}(\mathrm{c})}^{-}(\mathrm{y})\right\} .
\end{aligned}
$$

Hence $(F, A) \cap_{R}(G, B)$ is a bipolar fuzzy soft $\Gamma$. hypersubsemigroup of $\mathrm{S}$.

\section{Theorem 3.15}

Let $(F, A)$ and $(G, B)$ be two bipolar fuzzy soft $\Gamma$ -

hypersubsemigroup over $S$, then $(F, A) \cup_{R}(G, B)$ is a bipolar fuzzy soft -hypersub

semigroup of $\mathrm{S}$

Proof. Straight forward.

\section{Theorem 3.16}

Let $(F, A)$ and $(G, B)$ be two bipolar fuzzy soft

$\Gamma$-hyperbi(interior)ideal over $\mathrm{S}$, then $(\mathrm{F}, \mathrm{A}) \cap_{\mathrm{R}}(\mathrm{G}, \mathrm{B})$ is a bipolar fuzzy soft $\Gamma$-hyper

bi(interior)ideal of S.

Proof. Straight forward.

\section{Theorem 3.17}

Let $(F, A)$ and $(G, B)$ be two bipolar fuzzy soft $\Gamma$ hyperbi(interior)ideal over $\mathrm{S}$, then $(\mathrm{F}, \mathrm{A}) \cup_{\mathrm{R}}(\mathrm{G}, \mathrm{B})$ is a bipolar fuzzy soft $\Gamma$-hyperbi(interior)ideal of $S$.

Proof. Straight forward.

\section{Example 3.18}

Every bipolar fuzzy soft $\Gamma$-hyper ideal is bipolar valued fuzzy soft $\Gamma$-hypersubsemigroups but converse is not true.

Let $S=\{a, b, c, d, e\}$ and $\Gamma=\{\gamma\}$ then $S$ is $\Gamma$-semihypergroup

\begin{tabular}{|c|c|c|c|c|c|}
\hline$\gamma$ & $\mathrm{a}$ & $\mathrm{b}$ & $\mathrm{c}$ & $\mathrm{d}$ & $\mathrm{e}$ \\
\hline $\mathrm{a}$ & $\{\mathrm{a}, \mathrm{b}\}$ & $\{\mathrm{b}, \mathrm{e}\}$ & $\mathrm{c}$ & $\{\mathrm{c}, \mathrm{d}\}$ & $\mathrm{e}$ \\
\hline $\mathrm{b}$ & $\{\mathrm{b}, \mathrm{e}\}$ & $\mathrm{e}$ & $\mathrm{c}$ & $\{\mathrm{c}, \mathrm{d}\}$ & $\mathrm{e}$ \\
\hline $\mathrm{c}$ & $\mathrm{c}$ & $\mathrm{c}$ & $\mathrm{c}$ & $\mathrm{c}$ & $\mathrm{c}$ \\
\hline $\mathrm{d}$ & $\{\mathrm{c}, \mathrm{d}\}$ & $\{\mathrm{c}, \mathrm{d}\}$ & $\mathrm{c}$ & $\mathrm{d}$ & $\{\mathrm{c}, \mathrm{d}\}$ \\
\hline $\mathrm{e}$ & $\mathrm{e}$ & $\mathrm{e}$ & $\mathrm{c}$ & $\{\mathrm{c}, \mathrm{d}\}$ & $\mathrm{e}$ \\
\hline
\end{tabular}

Let $\mathrm{E}=\{\mathrm{u}, \mathrm{v}, \mathrm{w}, \mathrm{x}, \mathrm{y}\}$ and $\mathrm{A}=\{\mathrm{u}, \mathrm{v}, \mathrm{y}\}$. Define the bipolar fuzzy soft set $(F, A)$ as

$(\mathrm{F}, \mathrm{A})=\{\mathrm{F}(\mathrm{u}), \mathrm{F}(\mathrm{v}), \mathrm{F}(\mathrm{y})\}$, where

$\mathrm{F}(\mathrm{u})=\{(\mathrm{a}, 0.6,-0.5),(\mathrm{b}, 0.7,-0.6),(\mathrm{c}, 0.4,-0.2)$,

$(\mathrm{d}, 0.3,-0.1),(\mathrm{e}, 0.9,-0.8)\}$

$\mathrm{F}(\mathrm{v})=\{(\mathrm{a}, 0.8,-0.4),(\mathrm{b}, 0.9,-0.7),(\mathrm{c}, 0.6,-0.3)$

(d, $0.2,-0.1),(\mathrm{e}, 1,-0.9)\}$

$\mathrm{F}(\mathrm{y})=\{(\mathrm{a}, 0.7,-0.8),(\mathrm{b}, 0.8,-0.9),(\mathrm{c}, 0.5,-0.4)$,

(d, $0.2,-0.3),(e, 1,-0.9)\}$

Hence $(F, A)$ is a bipolar fuzzy soft sub $\Gamma$ - hypersemigroups but not bipolar valued fuzzy hyperideal. Sinc $\inf _{\mathrm{a} \in \mathrm{a} \gamma \mathrm{c}} \mu_{\mathrm{F}(\mathrm{a})}^{+}(\mathrm{a}) \geq$ $\max \left\{\mu_{\mathrm{F}(\mathrm{a})}^{+}(\mathrm{a}), \mu_{\mathrm{F}(\mathrm{a})}^{+}(\mathrm{c})\right\}$

$$
=0.4 \nsim 0.6
$$

\section{Example 3.19}

Every bipolar fuzzy soft $\Gamma$-hyperideal is bipolar valued fuzzy soft $\Gamma$ hyper bi-ideals but converse is not true.

Let $S=\{a, b, c, d, e\}$ and $\Gamma=\{\alpha, \beta\}$ then $S$ is $\Gamma$-hypersemigroup

\begin{tabular}{|c|c|c|c|c|c|}
\hline$\alpha$ & $\mathrm{a}$ & $\mathrm{b}$ & $\mathrm{c}$ & $\mathrm{d}$ & $\mathrm{e}$ \\
\hline $\mathrm{a}$ & $\{\mathrm{a}, \mathrm{b}\}$ & $\{\mathrm{b}, \mathrm{e}\}$ & $\mathrm{c}$ & $\{\mathrm{c}, \mathrm{d}\}$ & $\mathrm{e}$ \\
\hline $\mathrm{b}$ & $\{\mathrm{b}, \mathrm{e}\}$ & $\mathrm{e}$ & $\mathrm{c}$ & $\{\mathrm{c}, \mathrm{d}\}$ & $\mathrm{e}$ \\
\hline $\mathrm{c}$ & $\mathrm{c}$ & $\mathrm{c}$ & $\mathrm{c}$ & $\mathrm{c}$ & $\mathrm{c}$ \\
\hline $\mathrm{d}$ & $\{\mathrm{c}, \mathrm{d}\}$ & $\{\mathrm{c}, \mathrm{d}\}$ & $\mathrm{c}$ & $\mathrm{d}$ & $\{\mathrm{c}, \mathrm{d}\}$ \\
\hline $\mathrm{e}$ & $\mathrm{e}$ & $\mathrm{e}$ & $\mathrm{c}$ & $\{\mathrm{c}, \mathrm{d}\}$ & $\mathrm{e}$ \\
\hline
\end{tabular}

\begin{tabular}{|c|c|c|c|c|c|}
\hline$\beta$ & a & b & c & d & e \\
\hline a & $\{\mathrm{b}, \mathrm{e}\}$ & $\mathrm{e}$ & $\mathrm{c}$ & $\{\mathrm{c}, \mathrm{d}\}$ & $\mathrm{e}$ \\
\hline $\mathrm{b}$ & $\mathrm{e}$ & $\mathrm{e}$ & $\mathrm{c}$ & $\{\mathrm{c}, \mathrm{d}\}$ & $\mathrm{e}$ \\
\hline $\mathrm{c}$ & $\mathrm{c}$ & $\mathrm{c}$ & $\mathrm{c}$ & $\mathrm{c}$ & $\mathrm{c}$ \\
\hline $\mathrm{d}$ & $\{\mathrm{c}, \mathrm{d}\}$ & $\{\mathrm{c}, \mathrm{d}\}$ & $\mathrm{c}$ & $\mathrm{d}$ & $\{\mathrm{c}, \mathrm{d}\}$ \\
\hline $\mathrm{e}$ & $\mathrm{e}$ & $\mathrm{e}$ & $\mathrm{c}$ & $\{\mathrm{c}, \mathrm{d}\}$ & $\mathrm{e}$ \\
\hline
\end{tabular}

Let $\mathrm{E}=\{\mathrm{u}, \mathrm{v}, \mathrm{w}, \mathrm{x}, \mathrm{y}\}$ and $\mathrm{A}=\{\mathrm{w}, \mathrm{x}, \mathrm{y}\}$. Define the bipolar fuzzy soft set $(F, A)$ as

$(\mathrm{F}, \mathrm{A})=\{\mathrm{F}(\mathrm{w}), \mathrm{F}(\mathrm{x}), \mathrm{F}(\mathrm{y})\}$, where

$\mathrm{F}(\mathrm{w})=\{(\mathrm{a}, 0.2,-0.1),(\mathrm{b}, 0.4,-0.3),(\mathrm{c}, 1,-0.9)$,

(d, 0.6, -0.7), (e, 0.7, -0.8)\}

$\mathrm{F}(\mathrm{x})=\{(\mathrm{a}, 0.1,-0.2),(\mathrm{b}, 0.2,-0.3),(\mathrm{c}, 0.7,-0.8)$,

(d, $0.4,-0.5),(e, 0.5,-0.6)\}$ 
$\mathrm{F}(\mathrm{y})=\{(\mathrm{a}, 0.3,-0.1),(\mathrm{b}, 0.4,-0.2),(\mathrm{c}, 0.9,-0.7)$

$(\mathrm{d}, 0.6,-0.3),(\mathrm{e}, 0.8,-0.5)\}$

Hence $(F, A)$ is a bipolar fuzzy soft $\Gamma$-hyperbi-ideal but not bipolar valued fuzzy hyper ideal,

Since $\inf _{\mathrm{a} \in \mathrm{d} \alpha \mathrm{e}} \mu_{\mathrm{F}(\mathrm{a})}^{+}(\mathrm{a}) \geq \max \left\{\mu_{\mathrm{F}(\mathrm{a})}^{+}(\mathrm{d}), \mu_{\mathrm{F}(\mathrm{a})}^{+}(\mathrm{e})\right\}$ $=0.6 \nsucc 0.7$.

\section{Example 3.20}

Every bipolar fuzzy soft $\Gamma$-hyperideal is bipolar valued fuzzy soft $\Gamma-$ hyper intrior-ideal but converse is not true.

For the example 3.19, define the bipolar fuzzy soft set $(\mathrm{F}, \mathrm{A})$ as $(\mathrm{F}, \mathrm{A})=\{\mathrm{F}(\mathrm{w}), \mathrm{F}(\mathrm{x}), \mathrm{F}(\mathrm{y})\}$, where

$\mathrm{F}(\mathrm{w})=\{(\mathrm{a}, 0.3,-0.2),(\mathrm{b}, 0.6,-0.5),(\mathrm{c}, 0.9,-0.8)$,

(d, $0.2,-0.1),(\mathrm{e}, 0.8,-0.7)\}$

$\mathrm{F}(\mathrm{x})=\{(\mathrm{a}, 0.4,-0.3),(\mathrm{b}, 0.5,-0.4),(\mathrm{c}, 0.8,-0.7)$,

(d, $0.3,-0.1),(e, 0.6,-0.5)\}$

$\mathrm{F}(\mathrm{y})=\{(\mathrm{a}, 0.3,-0.2),(\mathrm{b}, 0.4,-0.5),(\mathrm{c}, 0.7,-0.9)$

(d, 0.2, -0.1), (e, 0.5, -0.8)\}

Hence $(F, A)$ is a bipolar fuzzy soft $\Gamma$-hyperinteriorideal but not bipolar valued fuzzy soft $\Gamma$-hyperideal, as $\inf _{\mathrm{a} \in \mathrm{b} \alpha \mathrm{d}} \mu_{\mathrm{F}(\mathrm{a})}^{+}(\mathrm{a}) \geq$ $\max \left\{\mu_{\mathrm{F}(\mathrm{a})}^{+}(\mathrm{b}), \mu_{\mathrm{F}(\mathrm{a})}^{+}(\mathrm{d})\right\}=0.2 \nsim 0.6$.

\section{Theorem 3.21}

Let $(\mathrm{F}, \mathrm{A})$ be a bipolar fuzzy soft set over S. (F, A) is a bipolar fuzzy soft $\Gamma$-hypersemigroup if and only if $(F, A)^{(t, s)}$ is a soft $\Gamma$-hypersemigroup of $S$ for each $t \in[0,1]$ and $s \in[-1,0]$.

Proof. Assume that $(\mathrm{F}, \mathrm{A})^{(\mathrm{t}, \mathrm{s})}$ is a bipolar soft $\Gamma$-hypersemigroup over $S$ for each $t \in[0,1]$ and $s \in[-1,0]$.For each $x_{1}, x_{2} \in S$ and $\mathrm{a} \in \mathrm{A} \quad$ let $\quad \mathrm{t}=\min \left\{\mu_{\mathrm{F}(\mathrm{a})}^{+}\left(\mathrm{x}_{1}\right), \mu_{\mathrm{F}(\mathrm{a})}^{+}\left(\mathrm{x}_{2}\right.\right.$ and $s=\max \left\{\mu_{\mathrm{F}(\mathrm{a})}^{-}\left(\mathrm{x}_{1}\right), \mu_{\mathrm{F}(\mathrm{a})}^{-}\left(\mathrm{x}_{2}\right)\right\}$, then $\mathrm{x}_{1}, \mathrm{x}_{2} \in \mu_{\mathrm{F}(\mathrm{a})}^{(\mathrm{t}, \mathrm{a})}$. Since $\mu_{\mathrm{F}(\mathrm{a})}^{(\mathrm{t}, \mathrm{s})}$ is a $\Gamma$ - hypersubsemigroup of $\mathrm{S}$, then $\mathrm{x}_{1}, \mathrm{x}_{2} \in \mu_{\mathrm{F}(\mathrm{a})}^{(\mathrm{t}) \mathrm{s}}$. That is $\mu_{\mathrm{F}(\mathrm{a})}^{+}\left(\mathrm{x}_{1} \gamma \mathrm{x}_{2}\right) \geq \mathrm{t}=\min \left\{\mu_{\mathrm{F}(\mathrm{a})}^{+}\left(\mathrm{x}_{1}\right), \mu_{\mathrm{F}(\mathrm{a})}^{+}\left(\mathrm{x}_{2}\right)\right\} \quad$ and $\mu_{\mathrm{F}(\mathrm{a})}^{-}\left(\mathrm{x}_{1} \gamma \mathrm{x}_{2}\right) \leq \mathrm{s}=\max \left\{\mu_{\mathrm{F}(\mathrm{a})}^{-}\left(\mathrm{x}_{1}\right), \mu_{\mathrm{F}(\mathrm{a})}^{-}\left(\mathrm{x}_{2}\right)\right\}$. This shows that $\mu_{\mathrm{F}(\mathrm{a})}$ is bipolar fuzzy $\Gamma$-hypersubsemigroup over $\mathrm{S}$. Thus $(\mathrm{F}, \mathrm{A})$ is a bipolar fuzzy soft $\Gamma$-hypersemigroup over $\mathrm{S}$.

Conversely, assume that $(F, A)$ is a bipolar fuzzy soft $\Gamma$-hypersemigroup. For each $a \in A, t \in[0,1]$ and $s \in[-1,0]$ and $\mathrm{x}_{1}, \mathrm{x}_{2} \in \mu_{\mathrm{F}(\mathrm{a})}^{(\mathrm{t})}$

we have $\mu_{\mathrm{F}(\mathrm{a})}^{+}\left(\mathrm{x}_{1}\right) \geq \mathrm{t}, \mu_{\mathrm{F}(\mathrm{a})}^{+}\left(\mathrm{x}_{2}\right) \geq \mathrm{t}$ and $\mu_{\mathrm{F}(\mathrm{a})}^{-}\left(\mathrm{x}_{1}\right) \leq \mathrm{s}$, $\mu_{\mathrm{F}(\mathrm{a})}^{-}\left(\mathrm{x}_{2}\right) \leq \mathrm{s}$. Therefore $\mu_{\mathrm{F}(\mathrm{a})}$ is a bipolar fuzzy $\Gamma$-hypersubsemigroup of S. Thus $\gamma \in \Gamma$ there exists $z \in x_{1} \gamma x_{2}$ such that

$\inf _{\mathrm{z} \in \mathrm{x}_{1} \gamma \mathrm{x}_{2}}(\mathrm{z}) \geq \min \left\{\mu_{\mathrm{F}(\mathrm{a})}^{+}\left(\mathrm{x}_{1}\right), \mu_{\mathrm{F}(\mathrm{a})}^{+}\left(\mathrm{x}_{2}\right)\right\} \geq \mathrm{t} \quad$ and $\sup _{\mathrm{z} \in \mathrm{x}_{1} \gamma \mathrm{x}_{2}}(\mathrm{z}) \leq$ $\max \left\{\mu_{\mathrm{F}(\mathrm{a})}^{-}\left(\mathrm{x}_{1}\right), \mu_{\mathrm{F}(\mathrm{a})}^{-}\left(\mathrm{x}_{2}\right)\right\} \leq \mathrm{s}$. Therefore for all $\mathrm{z} \in \mathrm{x}_{1} \gamma \mathrm{x}_{2}$ we have $\mathrm{z} \in \mu_{\mathrm{F}(\mathrm{a})}^{(\mathrm{t}, \mathrm{s})}$, this implies that $\mathrm{x}_{1} \gamma \mathrm{x}_{2} \in \mu_{\mathrm{F}(\mathrm{a})}^{(\mathrm{t})}$, that is $\mu_{\mathrm{F}(\mathrm{a})}^{(\mathrm{s})}$ is hyper $\Gamma$-subsemigroup of $\mathrm{S}$. Therefore $(\mathrm{F}, \mathrm{A})^{(\mathrm{t}, \mathrm{s})}$ is a soft $\Gamma$-hypersemigroup of $S$ for each $t \in[0,1]$ and $s \in[-1,0]$.

\section{Theorem 3.22}

Let $(F, A)$ be a bipolar fuzzy soft set over S. (F, A) is a bipolar fuzzy soft $\Gamma$-hyperleft(right)ideal if and only if $(F, A)^{(t, s)}$ is a soft $\Gamma$-hyper left(right) ideal of $S$ for each $t \in[0,1]$ and $s \in[-1,0]$. Proof. Suppose that $(F, A)^{(t, s)}$ is a bipolar soft $\Gamma$-hyperleftideal of $S$ for each $t \in[0,1], s \in[-1,0]$ and $a \in A, \gamma \in \Gamma$. For each $\mathrm{x}_{1} \in \mathrm{S}$, let $\mathrm{t}=\mu_{\mathrm{F}(\mathrm{a})}^{+}\left(\mathrm{x}_{1}\right)$, then $\mathrm{x}_{1} \in \mu_{\mathrm{F}(\mathrm{a})}^{(\mathrm{t}, \mathrm{s})}$. Since $\mu_{\mathrm{F}(\mathrm{a})}^{(\mathrm{t}, \mathrm{s})}$ is a $\Gamma$ hyper left ideal of $S$, then $x \gamma x_{1} \in \mu_{F(a)}^{(t, s)}$, for each $x \in S$. Hence $\mu_{\mathrm{F}(\mathrm{a})}^{+}\left(\mathrm{x} \mathrm{x}_{1}\right) \geq \mathrm{t}=\mu_{\mathrm{F}(\mathrm{a})}^{+}\left(\mathrm{x}_{1}\right)$ and $\mu_{\mathrm{F}(\mathrm{a})}^{-}\left(\mathrm{x} \mathrm{x}_{1}\right) \leq \mathrm{s}=\mu_{\mathrm{F}(\mathrm{a})}^{-}\left(\mathrm{x}_{1}\right)$. This shows that $\mu_{\mathrm{F}(\mathrm{a})}$ is bipolar fuzzy $\Gamma$-hyperleftideal of $\mathrm{S}$. By definition 3.2, $(\mathrm{F}, \mathrm{A})$ is a bipolar fuzzy soft $\Gamma$-hyperleftideal of $\mathrm{S}$. Conversely, assume that $(F, A)$ is a bipolar fuzzy soft $\Gamma$-hyper left ideal of $S$. For each $a \in A, t \in[0,1]$ and $s \in[-1,0]$ and $x_{1} \in$ $\mu_{\mathrm{F}(\mathrm{a})}^{(\mathrm{t}, \mathrm{s})}$ we have $\mu_{\mathrm{F}(\mathrm{a})}^{+}\left(\mathrm{x}_{1}\right) \geq \mathrm{t}$, and $\mu_{\mathrm{F}(\mathrm{a})}^{-}\left(\mathrm{x}_{1}\right) \leq \mathrm{s}$ and by definition
3.2, $\mu_{\mathrm{F}(\mathrm{a})}^{+}$and $\mu_{\mathrm{F}(\mathrm{a})}^{-}$is a bipolar fuzzy $\Gamma$-hyper left ideal of $\mathrm{S}$. Thus for $\gamma \in \Gamma$ there exists $\mathrm{z} \in \mathrm{x} \gamma \mathrm{x}_{1}$ such that $\inf _{\mathrm{z} \in \mathrm{x}_{\mathrm{x}}}(\mathrm{z}) \geq$ $\mu_{\mathrm{F}(\mathrm{a})}^{+}\left(\mathrm{x}_{1}\right) \geq \mathrm{t}$ and $\sup _{(\mathrm{z})} \leq \mu_{\mathrm{F}(\mathrm{a})}^{-}\left(\mathrm{x}_{1}\right) \leq \mathrm{s}$. Therefore for all $\mathrm{z} \in \mathrm{x} \gamma \mathrm{x}_{1}$ we have $\mathrm{z} \in \mu_{\mathrm{F}(\mathrm{a})}^{(\mathrm{t}, \mathrm{s})}$, that is $\mu_{\mathrm{F}(\mathrm{a})}^{(\mathrm{t}, \mathrm{s})}$ is hyper $\Gamma$-left ideal of $\mathrm{S}$. Therefore $(\mathrm{F}, \mathrm{A})^{(\mathrm{t}, \mathrm{s})}$ is a soft $\Gamma$-hyper left ideal of $\mathrm{S}$ for each $t \in[0,1]$ and $s \in[-1,0]$. Similar proof holds for right ideal also.

\section{Theorem3.23}

Let $(\mathrm{F}, \mathrm{A})$ be a bipolar fuzzy soft set over $\mathrm{S},(\mathrm{F}, \mathrm{A})$ is a bipolar fuzzy soft $\Gamma$-hyperideal if and only if $(F, A)^{(t, s)}$ is a soft

$\Gamma$-hyperideal of $S$ for each $t \in[0,1]$ and $s \in[-1,0]$.

Proof. The proof follows from theroem 3.22

\section{Bipolar Fuzzy Soft Image and Inverse Image of Hyper $\Gamma$-Semigroups}

\section{Definition 4.1}

[9] Let $\eta: \mathrm{H}_{1} \rightarrow \mathrm{H}_{2}$ and $\psi: \mathrm{A} \rightarrow \mathrm{B}$ be two functions, $\mathrm{A}$ and $\mathrm{B}$ be two parametric sets from the crisp sets $\mathrm{H}_{1}$ and

$\mathrm{H}_{2}$, respectively. Then the pair $(\eta, \psi)$ is called a bipolar fuzzy soft function from $\mathrm{H}_{1}$ to $\mathrm{H}_{1}$.

\section{Definition 4.2}

Let $(F, A)$ and $(G, B)$ be two bipolar fuzzy soft sets over the sets $\mathrm{H}_{1}$ and $\mathrm{H}_{2}$, respectively, and $(\eta, \psi)$ be a bipolar fuzzy soft map from $\mathrm{H}_{1}$ to $\mathrm{H}_{2}$.

(i) The image of $(F, A)$ under $(\eta, \psi)$ denoted by $(\eta, \psi)(F, A)$, is a bipolar fuzzy soft set over $\mathrm{H}_{2}$ defined by $(\eta, \psi)(\mathrm{F}, \mathrm{A})=$

( $\eta(F), \psi(A)$ ), where for all $b \in \psi(A)$ and for all $y \in \mathrm{H}_{2}$,

$$
\begin{aligned}
& \mu_{\eta_{F(b)}}^{+}(y)=\left\{\begin{array}{cl}
\sup _{\eta(x)=y \psi(a)=b} \sup _{F(a)} \mu^{+}(x), & \operatorname{iff}^{-1}(y) \neq \phi \\
0 & \text { otherwise }
\end{array}\right. \\
& \mu_{\eta_{\mathrm{F}(\mathrm{b})}}^{-}(y)= \begin{cases}\inf _{\eta(\mathrm{x})=\mathrm{y} \psi(\mathrm{a})=\mathrm{b}} \inf _{\mathrm{F}(\mathrm{a})}(\mathrm{x}), & \mathrm{iff}^{-1}(\mathrm{y}) \neq \phi \\
0 & \text { otherwise }\end{cases}
\end{aligned}
$$

(ii) The inverse image of $(G, B)$ under $(\eta, \psi)$ denoted by $(\eta, \psi)^{-1}(G, B)$, is a bipolar fuzzy soft set over $H_{1}$ defined by $(\eta, \psi)^{-1}(G, B)=\left(\eta^{-1}(G), \psi^{-1}(B)\right)$, where for all a $\in \psi^{-1}(B)$ and for all $\mathrm{x} \in \mathrm{H}_{1}, \mu_{\eta_{\mathrm{G}(\mathrm{a})}^{-1}}^{+}(\mathrm{y})=\mu_{\mathrm{G}_{\Psi(\mathrm{a})}}^{+}(\eta(\mathrm{x}))$ and $\mu_{\eta_{\mathrm{G}(\mathrm{a})}^{-1}}^{-}(\mathrm{y})=$ $\mu_{\mathrm{G}_{\Psi(a)}}(\eta(\mathrm{x}))$

\section{Theorem 4.3}

Let $\eta: \mathrm{H}_{1} \rightarrow \mathrm{H}_{2}$ be a homomorphism of S. If $(G, B)$ is a bipolar fuzzy soft $\Gamma$-hypersubsemigroup of $\mathrm{H}_{2}$, then $(\eta, \psi)^{-1}(\mathrm{G}, \mathrm{B})$ is a bipolar fuzzy soft $\Gamma$-hypersubsemigroup of $\mathrm{H}_{1}$.

Proof. Let $(\mathrm{G}, \mathrm{B})$ is a bipolar fuzzy soft $\Gamma$-hypersubsemigroup of $\mathrm{H}_{2}$. Let $\mathrm{x}, \mathrm{y}, \mathrm{z} \in \mathrm{H}_{1}, \gamma \in \Gamma_{1}$ then we have

$$
\begin{aligned}
& \inf _{\mathbf{z} \in \mathbf{X} Y \mathbf{y}}\left\{\boldsymbol{\mu}_{\boldsymbol{\eta}_{\mathbf{G}(\mathbf{a})}^{-1}}^{+}(\mathbf{z})\right\}=\inf _{\mathbf{z} \in \mathbf{X Y Y}}\left\{\boldsymbol{\mu}_{\mathbf{g}_{\Psi(a)}^{+}}^{+}(\boldsymbol{\eta}(\mathbf{z}))\right\} \\
& =\inf _{\boldsymbol{\eta}(\mathbf{z}) \in \mathfrak{\eta}(\mathbf{x y y})}\left\{\boldsymbol{\mu}_{\mathrm{g} \psi(\mathbf{a})}^{+}(\boldsymbol{\eta}(\mathbf{z}))\right\} \\
& =\inf _{\eta(\mathbf{z}) \in \boldsymbol{\eta}(\mathbf{x}) \mathbf{h}(\boldsymbol{\gamma}) \boldsymbol{\eta}(\mathbf{y})}\left\{\boldsymbol{\mu}_{\mathrm{g}_{\psi}(\mathbf{a})}^{+}(\boldsymbol{\eta}(\mathbf{z}))\right\} \\
& \geq \min \left\{\boldsymbol{\mu}_{\mathrm{g}_{\psi(a)}}^{+} \boldsymbol{\eta}(\mathbf{x}), \boldsymbol{\mu}_{\mathrm{g}_{\psi(a)}}^{+} \boldsymbol{\eta}(\mathbf{y})\right\} \\
& =\min \left\{\boldsymbol{\mu}_{\boldsymbol{\eta}_{\mathrm{G}(\mathrm{a})}^{-1}}^{+}(\mathbf{x}), \boldsymbol{\mu}_{\boldsymbol{\eta}_{\mathrm{G}(\mathrm{a})}^{-1}}^{+}(\mathrm{y})\right\} \\
& \sup _{\mathbf{z} \in \mathbf{x} Y \mathbf{y}}\left\{\boldsymbol{\mu}_{\eta_{\mathbf{G}(a)}^{-}(\mathbf{z})}^{-}\right\}=\sup _{\mathbf{z} \in \mathbf{X} Y \mathbf{y}}\left\{\boldsymbol{\mu}_{\mathbf{g}_{\Psi(a)}^{-}}^{-}(\boldsymbol{\eta}(\mathbf{z}))\right\} \\
& =\sup _{\eta(z) \in \eta(x \gamma y)}\left\{\boldsymbol{\mu}_{\mathbf{z}_{\psi(a)}}^{-}(\boldsymbol{\eta}(\mathbf{z}))\right\} \\
& =\sup _{\eta(\mathbf{z}) \in \eta(\mathrm{x}) \mathrm{h}(\gamma) \eta(\mathbf{y})}\left\{\boldsymbol{\mu}_{\mathrm{g}_{\psi}(\mathbf{a})}^{-}(\boldsymbol{\eta}(\mathbf{z}))\right\} \\
& \leq \max \left\{\boldsymbol{\mu}_{\mathrm{g}_{\psi(a)}}^{-} \eta(\mathrm{x}), \boldsymbol{\mu}_{\mathrm{g} \psi(\mathrm{a})}^{-} \eta(\mathrm{y})\right\} \\
& =\max \left\{\boldsymbol{\mu}_{\eta_{\mathbf{G}(a)}^{-1}}^{-}(x), \boldsymbol{\mu}_{\eta_{G(a)}^{-1}}^{-}(y)\right\}
\end{aligned}
$$


Therefore $(\eta, \psi)^{-1}(G, B)$ is a bipolar fuzzy soft $\Gamma$-hypersubsemigroup of $\mathrm{H}_{1}$.

\section{Theorem 4.4}

Let $\eta: \mathrm{H}_{1} \rightarrow \mathrm{H}_{2}$ be a homomorphism of S. If $(G, B)$ is a bipolar fuzzy soft $\Gamma$-hyperleft(right, bi-ideal, interior) of $\mathrm{H}_{2}$, then $(\eta, \psi)^{-1}(G, B)$ is a bipolar fuzzy soft $\Gamma$-hyperleft(right, bi-ideal, interior)ideal of $\mathrm{H}_{1}$.

Proof. Straightforward.

\section{Theorem4.5}

Let $\eta: \mathrm{H}_{1} \rightarrow \mathrm{H}_{2}$ be a homomorphism of $\mathrm{S}$. If $(\mathrm{F}, \mathrm{A})$ is a bipolar fuzzy soft $\Gamma$-hypersubsemigroup of $\mathrm{H}_{1}$, then $(\eta, \psi)(\mathrm{F}, \mathrm{A})$ is a bipolar fuzzy soft $\Gamma$-hypersubsemigroup of $\mathrm{H}_{2}$.

Proof. Let $(F, A)$ is a bipolar fuzzy soft

$\Gamma$-hypersubsemigroup of $\mathrm{H}_{1}$. Let $\mathrm{x}_{1}, \mathrm{y}_{1} \mathrm{z}_{1} \in \mathrm{H}_{2}, \gamma \in \Gamma_{2}$ then we have

$$
\begin{aligned}
& \inf _{\mathrm{z}_{1} \in \mathrm{X}_{1} Y \mathrm{Y}_{1}}\left\{\mu_{\eta_{\mathrm{F}}(\mathrm{b})}^{+}\left(\mathrm{z}_{1}\right)\right\}=\inf _{\mathrm{z}_{1} \in \mathrm{x}_{1} Y \mathrm{y}_{1}}\left\{\sup _{\mathrm{t} \in \eta^{-1}\left(\mathrm{z}_{1}\right) \psi(\mathrm{a})=\mathrm{b}} \sup _{\mathrm{F}(\mathrm{a})}(\mathrm{t})\right\} \\
& \geq \inf _{\mathrm{z} \in \mathrm{X}_{1} Y \mathrm{Y}_{1}}\left\{\sup _{\psi(\mathrm{a})=\mathrm{b}} \mu_{\mathrm{F}}^{+}(\mathrm{b})\left(\mathrm{z}_{1}\right)\right\} \\
& =\inf _{\eta(\mathrm{z}) \in \eta(\mathrm{x}) \mathrm{h}(\gamma) \eta(\mathrm{y})}\left\{\sup _{\psi(\mathrm{a})=\mathrm{b}} \mu_{\mathrm{F}(\mathrm{b})}^{+}(\mathrm{z})\right\} \\
& =\inf _{\eta(\mathrm{z}) \in \eta(\mathrm{x} Y \mathrm{y})}\left\{\sup _{\psi(\mathrm{a})=\mathrm{b}} \mu_{\mathrm{F}(\mathrm{b})}^{+}(\mathrm{z})\right\} \\
& =\inf _{\mathrm{z} \in \mathrm{XXY}}\left\{\sup _{\psi(\mathrm{a})=\mathrm{b}} \mu_{\mathrm{F}}^{+}(\mathrm{b})(\mathrm{z})\right\} \\
& \geq \sup _{\psi(\mathrm{a})=\mathrm{b}} \min \left\{\mu_{\mathrm{F}(\mathrm{b})}^{+}(\mathrm{x}), \mu_{\mathrm{F}(\mathrm{b})}^{+}(\mathrm{y})\right\} \\
& \geq \sup _{\mathrm{x} \gamma \mathrm{y} \subseteq \eta^{-1}\left(\mathrm{x}_{1}\right) \mathrm{h}^{-1}(\gamma) \eta^{-1}\left(\mathrm{y}_{1}\right)}\left\{\sup _{\Psi(\mathrm{a})=\mathrm{b}} \min \left\{\mu_{\mathrm{F}(\mathrm{b})}^{+}(\mathrm{x}), \mu_{\mathrm{F}(\mathrm{b})}^{+}(\mathrm{y})\right\}\right\} \\
& =\min \left\{\sup _{\eta(x)=y \psi(a)=b} \sup _{F(a)} \mu^{+}(x), \sup _{\eta(x)=y \psi(a)=b} \sup _{F(a)} \mu^{+}(y)\right\} \\
& \geq \min \left\{\mu_{\eta_{\mathrm{F}(\mathfrak{b})}}^{+}\left(\mathrm{x}_{1}\right), \mu_{\eta_{\mathrm{F}(\mathfrak{b})}}^{+}\left(\mathrm{y}_{1}\right)\right\} \\
& \sup _{\mathrm{z}_{1} \in \mathrm{x}_{1} Y \mathrm{y}_{1}}\left\{\mu_{\eta_{\mathrm{F}(\mathrm{b})}}^{-}\left(\mathrm{z}_{1}\right)\right\}=\sup _{\mathrm{z}_{1} \in \mathrm{x}_{1} \gamma \mathrm{y}_{1}}\left\{\inf _{\mathrm{t} \in \eta^{-1}\left(\mathrm{z}_{1}\right) \psi(\mathrm{a})=\mathrm{b}} \mu_{\mathrm{F}(\mathrm{a})}^{-}(\mathrm{t})\right\} \\
& \leq \sup _{\mathrm{z} \in \mathrm{x}_{1} \gamma \mathrm{y}_{1}}\left\{\inf _{\Psi(\mathrm{a})=\mathrm{b}} \mu_{\mathrm{F}(\mathrm{b})}^{-}\left(\mathrm{z}_{1}\right)\right\} \\
& =\sup _{\eta(\mathrm{z}) \in \eta(\mathrm{x}) \mathrm{h}(\gamma) \eta(\mathrm{y})}\left\{\inf _{\psi(\mathrm{a})=\mathrm{b}} \mu_{\mathrm{F}(\mathrm{b})}^{-}(\mathrm{z})\right\} \\
& =\sup _{\eta(\mathrm{z}) \in \eta(\mathrm{x} \gamma \mathrm{y})}\left\{\inf _{\psi(\mathrm{a})=\mathrm{b}} \mu_{\mathrm{F}(\mathrm{b})}^{-}(\mathrm{z})\right\} \\
& =\sup _{\mathrm{z} \in \mathrm{x} Y \mathrm{y}}\left\{\inf _{\psi(\mathrm{a})=\mathrm{b}} \mu_{\mathrm{F}(\mathrm{b})}^{-}(\mathrm{z})\right\} \\
& \leq \inf _{\psi(\mathrm{a})=\mathrm{b}} \max \left\{\mu_{\mathrm{F}(\mathrm{b})}^{-}(\mathrm{x}), \mu_{\mathrm{F}(\mathrm{b})}^{-}(\mathrm{y})\right\} \\
& \leq \inf _{x y y \subseteq \eta^{-1}\left(x_{1}\right) h^{-1}(\gamma) \eta^{-1}\left(y_{1}\right)}\left\{\inf _{\psi(a)=\mathrm{b}} \max \left\{\mu_{\mathrm{F}(\mathrm{b})}^{-}(\mathrm{x}), \mu_{\mathrm{F}}^{-}(\mathrm{b})(\mathrm{y})\right\}\right\} \\
& =\max \left\{\inf _{\eta(\mathrm{x})=y \psi(\mathrm{a})=\mathrm{b}} \inf _{\mathrm{F}(\mathrm{a})}^{-}(\mathrm{x}), \inf _{\eta(\mathrm{x})=\mathrm{y} \psi(\mathrm{a})=\mathrm{b}} \inf _{\mathrm{F}(\mathrm{a})}^{-}(\mathrm{y})\right\} \\
& \leq \max \left\{\mu_{\eta_{\mathrm{F}(\mathfrak{b})}}^{-}\left(\mathrm{x}_{1}\right), \mu_{\eta_{\mathrm{F}(\mathfrak{b})}^{-}}^{-}\left(\mathrm{y}_{1}\right)\right\}
\end{aligned}
$$

Therefore $(\eta, \psi)(F, A)$ is a bipolar fuzzy soft $\Gamma$-hyper subsemigroup of $\mathrm{H}_{2}$.

\section{Theorem 4.6}

Let $\eta: \mathrm{H}_{1} \rightarrow \mathrm{H}_{2}$ be a homomorphism of S. If $(F, A)$ is a bipolar fuzzy soft $\Gamma$-hyperleft(right, bi-ideal, interior)ideal of $\mathrm{H}_{1}$, then $(\eta, \psi)(F, A)$ is a bipolar fuzzy soft $\Gamma$-hyperleft(right, bi-ideal, interior)ideal of $\mathrm{H}_{2}$.

Proof. Straighforward.

\section{References}

[1] S. M. Anvariyeh, S. Miravakili and B. Davvaz, On $\Gamma$-hyperideals in $\Gamma$ - hypersemigroups, Carpathian Journal of Mathematics 26(1) (2010), 11-23.

[2] M. Aslam, S. Abdullah and K.Ullah, Biploar fuzzy sets and its application in decision making problem, arXiv, 1303.6932v1[cs.AI], 2013.

[3] Aygunoglu and H. Aygun, Introduction to fuzzy soft groups, Comput.Math. Appl.58(2009) 1279-1286.
[4] K. M. Lee , Bi-polar-valued fuzzy sets and their operations, Proc Int Conf Intelligent Technologies Bangkok, Thailand,(2000) 307-12.

[5] K. M. Lee. Comparasion of interval valued fuzzy sets, intuitionistiv fuzzy sets, and bi-polar-valued fuzzy sets. J. Fuzzy Logic Intel Syst. 200414 125-9

[6] P. K. Maji,R. Biswas and R. Roy, Fuzzy soft sets, J Fuzzy Math. Appl,9(3) (2001)589-602.

[7] F. Marty, Sur une generalization de la notion de group, in. proc 8th Congress Mathematics Scandenaves, Stockholm, 1994, 45-49.

[8] D. Molodtsov, Soft set theory first results, Comput. Math. Appl, 37 (1999)19-31.

[9] Muhammad Akram, Bipolar fuzzy soft Lie algebras,Quasigroups and related systems 21 (2013) 1-10.

[10] Muhammad Akram, J. Kavikumar and Azme Bin Khamis, Fuzzy soft $\Gamma$-semigroups, Appl.Math. Inf. Sci 8(2)(2014) 929-934.

[11] Muhammad Akram, J. Kavikumar and Azme Bin Khamis, Characterization pf bipolar fuzzy soft $\Gamma$-semigroups, Indian Journal of Science and Technology 7(8)(2014) 1211-1221.

[12] Munazza Naz, Muhammad Shabir and Muhammad Irfan Ali, On Fuzzy Soft Semigroups,World Appl. Sci 22 (2013)62-83.

[13] Naveed Yaqoob and Moin A. Ansari, Bipolar $(\lambda, \delta)$-Fuzzy ideals in Ternary semigroups, Int. Journal of Math. Analysis 7 (36)(2013)1775-1782.

[14] Ghareeb,Structures of bipolar fuzzy $\Gamma$-hyperideals in $\Gamma$-semihypergroups, Journal of intelligent and fuzzy systems 27 (2014) 3015-3032.

[15] S. Onar, B.A.Ersoy and U. Tekir, Fuzzy soft $\Gamma$-ring, Iranian Journal of Science and technology, A4 (2012) 469-476.

[16] M. K. Sen and N. K Saha, On $\Gamma$-semigroup, I, Bull.Calcutta Math Soc., 78 180-186 (1986)

[17] Violeta Leoreanu-Fotea, Feng Feng and Jianming Zhan,Fuzzy soft hypergroups, International Journal of Computer Mathematics. 89(8) (2012)963-974.

[18] L. A Zadeh, Fuzzy sets. Information and control. 8 (1965) 338-353.

[19] Zhang WR. Bipolar fuzzy sets, Proceedings of FUZZ-IEEE, (1998) 835-840. 